\title{
Trimetazidina na Injúria de Isquemia e Reperfusão em Cirurgia de Revascularização do Miocárdio
}

\author{
Trimetazidine on Ischemic Injury and Reperfusion in Coronary Artery Bypass Grafting

\begin{abstract}
Gerez Fernandes Martins ${ }^{1}$, Aristarco Gonçalves de Siqueira Filho ${ }^{2}$, João Bosco de Figueiredo Santos ${ }^{1}$, Claudio Roberto Cavalcanti Assunção ${ }^{1}$, Francisca Bottino¹, Káttia Gerúndio de Carvalho , Alberto Valência

Instituto Estadual de Cardiologia Aloysio de Castro; Universidade Federal do Rio de Janeiro², Rio de Janeiro, RJ - Brasil
\end{abstract}

\section{Resumo}

Fundamento: A injúria de isquemia e reperfusão constitui um mecanismo fisiopatológico frequente e de difícil controle durante a Cirurgia de Revascularização do Miocárdio (CRVM) com circulação extracorpórea, sendo o momento crítico o término da cirurgia, quando ocorre o desclampeamento da aorta e a liberação dos radicais hiperóxidos causadores da injúria.

Objetivo: Avaliar, em estudo prospectivo, duplo-cego randomizado, controlado com placebo, os efeitos da Trimetazidina (Tmz) sobre a injúria de isquemia e reperfusão miocárdica, identificando a variação dos marcadores plasmáticos de agressão miocárdica (troponina T e CPK-Mb), e as alterações ecocardiográficas da função ventricular.

Métodos: Foram estudados 60 pacientes, divididos em dois grupos (Placebo e Tmz) com, no máximo, disfunção ventricular leve, estratificados por ecocardiografia e recebendo medicação/placebo na dose - no pré-operatório sem medicação, 12 a 15 dias de medicação/placebo colhida cinco minutos após o desclampeamento aórtico, e nas 12, 24 e 48 horas seguintes.

Resultados: Tanto a troponina T como a CPK-Mb atingiram valores altamente significativos $(p=0,0001)$ no grupo tratado em relação ao grupo controle nos quatro momentos analisados - $5 \mathrm{~min}, 12 \mathrm{~h}, 24 \mathrm{~h}$ e 48 h. As variáveis ecocardiográficas não evidenciaram mudanças evolutivas em cada grupo isoladamente e quando comparados em conjunto.

Conclusão: A trimetazidina mostrou-se eficaz na redução da injúria de isquemia e reperfusão, não interferiu na função ventricular esquerda, e não foram observados efeitos colaterais. (Arq Bras Cardiol 2011; 97(3) : 209-216)

Palavras-chave: Trimetazidina/administração e dosagem, reperfusão miocárdica, isquemia miocárdica, revascularização miocárdica.

\footnotetext{
Abstract

Background: The ischemia and reperfusion ischemia is a common physiopathological mechanisms, which has difficult control during Coronary Artery Bypass Grafting (CABG) with cardiopulmonary bypass, the critical moment of which happening by the end of surgery, when there is declamping of aorta and release of hyperoxic radicals causing the injury.
}

Objective: Evaluate, in a randomized double-blind prospective study, controlled with placebo, the effects of Trimetazidine (Tmz) on ischemic injury and myocardial reperfusion, identifying the change in plasma markers of a myocardial aggression (troponin T and CPK-MB), and echocardiographic changes of ventricular function.

Methods: We studied 60 patients divided in two groups (placebo and Tmz) with mild ventricular dysfunction at the most, stratified by echocardiography and receiving medication/placebo at a dose of $20 \mathrm{mg} / 3 \mathrm{x} /$ day, starting from 12 to 15 days after pre-operative period up to 5 to 8 days after post-operative period. Troponin T and CPK-Mb were measured preoperatively without medication, 12 to 15 days of medication/ placebo taken five minutes after aortic declamping, and at subsequent 12, 24 and 48 hours.

Results: Both Troponin T and CPK-Mb reached highly significant values $(p=0.0001)$ in the treated group compared to the control group at the four moments analyzed - $5 \mathrm{~min}, 12 \mathrm{~h}, 24 \mathrm{~h}$ and $48 \mathrm{~h}$. The echocardiographic variables did not show evolutive changes in each group severally considered and when compared among themselves.

Conclusion: : Trimetazidine was effective in reducing ischemic injury and reperfusion, had no effect on left ventricular function, and no side effects were observed. (Arq Bras Cardiol 2011; 97(3) : 209-216)

Keywords: Trimetazidine/administration \& dosage; myocardial reperfusion; myocardial ischemia; myocardial revascularization.

Full texts in English - http://www.arquivosonline.com.br

Correspondência: Gerez Fernandes Martins •

Rua Humaitá, 231 / 101 - Humaitá - 22261-000 - Rio de Janeiro, RJ - Brasil

E-mail: gerezm@cardiol.br, gerezm@terra.com.br

Artigo recebido em 16/11/10; revisado recebido em 02/03/11; aceito em 11/04/11. 


\section{Introdução}

A injúria de reperfusão é um fenômeno fisiopatológico que pode ocorrer nas síndromes coronarianas em geral, como na angina instável, angina vasoespástica, infarto sem supra desnivelamento e infarto do miocárdio, seguido ou não de procedimentos como trombólise ou angioplastia, assim como nas cirurgias cardiovasculares e nas angioplastias eletivas'.

O fenômeno vem chamando a atenção de pesquisadores por suas evidentes e significativas repercussões na prática clinicocardiológica e tem levado a uma melhor compreensão dos eventos a ele relacionados, em particular a partir dos chamados radicais hiperóxidos, formados ao final do metabolismo oxidativo celular, que podem causar a injúria após o desclampeamento da aorta na cirurgia cardíaca. Os radicais hiperóxidos compreendem o ânion superóxido $\left(\mathrm{O}^{-2}\right)$, o radical hidroxila $(\mathrm{OH})$ e o peróxido de hidrogênio $\left(\mathrm{H}^{2} \mathrm{O}^{2}\right)$, as quais são removidos das células por sistemas de enzimas com funções antioxidantes, normalmente presentes na fisiologia miocárdica. Esses sistemas de enzimas endógenas com ação antioxidante são responsáveis pela limitação do acúmulo

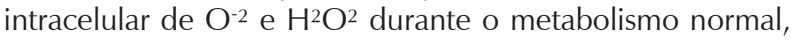
reduzindo o dano oxidativo sobre proteínas e lipídeos ${ }^{2-4}$.

Para melhorar a proteção miocárdica durante procedimentos que envolvam injúria de reperfusão, recentemente as atenções voltaram-se para a pesquisa de drogas citoprotetoras com ação em segmentos do metabolismo celular, utilizadas isoladamente ou em associação para reduzir ou prevenir o dano sobre a célula cardíaca ${ }^{5,6}$.

O mecanismo de ação citoprotetor da Tmz nas alterações do metabolismo cardíaco durante a isquemia abriu caminho para uma abordagem terapêutica na reperfusão. Com sua ação anti-isquêmica, reduz os danos metabólicos provocados durante a isquemia por agir numa etapa crítica do metabolismo cardíaco, bloqueando a betaoxidação dos ácidos graxos, mediante a inibição da 3-acetil-CoA tiolase de cadeia longa. Esse efeito anti-isquêmico resulta no aumento da oxidação da glicose, adicional à glicólise, tendo como consequência a redução de prótons, elevação do pH intracelular e da acidose tecidual, recuperação da eficiência cardíaca e melhoria da produção de acetil-CoA. Além desses efeitos, limita o acúmulo de cálcio, a inflamação e a produção de radicais livres hiperóxidos que ocorrem após reperfusão, sem provocar alterações hemodinâmicas. Sua utilização na profilaxia da disfunção ventricular tem demonstrado também ação benéfica sobre o "miocárdio atordoado", tradução clínica da injúria sobre a função ventricular após CRVM ${ }^{7-10}$.

Este estudo avalia efeitos de melhora e proteção ao miocárdio reperfundido da substância trimetazidina (Tmz), utilizada como pré-tratamento em pacientes submetidos a CRVM com circulação extracopórea, sobre a injúria de isquemia e reperfusão miocárdica, a partir da variação dos marcadores plasmáticos de agressão miocárdica (troponina T e CPK-Mb) e de alterações ecocardiográficas da função ventricular ${ }^{11,12}$.

\section{Métodos}

Ensaio clínico prospectivo, duplo cego, randomizado e controlado por placebo, realizado entre julho de 2007 e agosto de 2008, no Instituto de Cardiologia Aloysio de Castro, Rio de Janeiro (IECAC-RJ). Tendo como objetivo principal avaliar a diferença significativa nos marcadores sorológicos de injuria miocárdica e nos parâmetros ecocardiográficos entre dois grupos submetidos a CRVM, grupo tratado e grupo placebo.

Foram consideradas as seguintes suposições para o cálculo do tamanho da amostra: nível de significância de $5 \%$; poder do teste estatístico de $80 \%$; e diferença esperada nos marcadores relativamente "grande" entre os grupos, essa conhecida como tamanho do efeito, obtido por conhecimento prévio da literatura. De acordo com Cohen $^{13}$, o número de casos necessários para cada grupo analisado é de 28 indivíduos, devendo-se considerar uma perda máxima ao longo do estudo de $30 \%$. Assim, uma amostra contendo dois grupos deveria incluir 73 pacientes.

Foram avaliados clinicamente 137 pacientes coronariopatas com indicação de CRVM, sendo inicialmente selecionados 75 mediante protocolo ecocardiográfico transtorácico que utilizou a análise da contração segmentar para classificar a função ventricular por meio do cálculo do índice de contração segmentar (ICS), por 17 segmentos $^{14}$. Foram excluídos os pacientes com disfunção moderada e grave, permanecendo apenas aqueles com função ventricular normal (ICS =1) ou com disfunção sistólica leve (ICS = 1,1 a 1,6). A avaliação dividiu os pacientes em dois subgrupos: sem alteração na contração segmentar e com alteração na contração segmentar, daqui em diante grupo SAS e grupo CAS, respectivamente, com objetivo de analisar a uniformidade entre os grupos placebo e Tmz. Foram incluídos os pacientes que necessitassem unicamente de CRVM com circulação extracorpórea e que concordassem em assinar o termo de consentimento.

Dos 75 pacientes selecionados, três desistiram da cirurgia, dois operaram em outra instituição, dois não utilizaram a medicação, quatro descontinuaram com 24 horas a medicação e quatro necessitaram de reoperação por sangramento evoluindo para óbito. Portanto, 60 pacientes efetivamente concluíram o estudo sem intercorrências clínicas ou cirúrgicas, sendo portadores de lesões corrigidas em três ou mais ramos coronarianos.

Os pacientes foram submetidos a três avaliações ecocardiográficas durante a pesquisa. A primeira avaliação (D0) foi utilizada como critério de inclusão através do ICS, e as duas subsequentes (D1 e D2) como seguimento do estudo. Foram também realizadas análises da função ventricular pelo método de Simpson com monitorização eletrocardiográfica, padronizando os momentos da imagem em sístole (pelo pico da onda R do eletrocardiograma) e diástole (pelo final da onda T do eletrocardiograma), e pelo índice de TEI (relação entre sístole e diástole).

A primeira avaliação ecocardiográfica (D0) foi feita nos pacientes sem utilização de medicação; a seguinte (D1), no dia da cirurgia, estando todos em uso de no mínimo 12 e no máximo 15 dias de medicação/placebo; e a terceira (D2), na alta da UTI, entre o terceiro e o quinto dias do pós-operatório, utilizando a medicação/placebo entre 15 e 18 dias.

A Tmz foi fornecida aos pacientes após mascaramento com randomização na proporção 1:1, permutada em blocos pelo programa Cytools Excel add-in. Os avaliadores não tinham 
conhecimento sobre quais pacientes haviam utilizado droga ou placebo. Os estudos estatísticos foram realizados também de forma cega, com os códigos referentes ao medicamento/ placebo quebrados apenas após a análise estatística.

Os pacientes elegíveis receberam a dose de $60 \mathrm{mg}$ da medicação/placebo divididas em três tomadas diárias, iniciadas com mínimo 12 e máximo 15 dias precedentes ao ato cirúrgico, tendo os 60 pacientes permanecido com a droga por um intervalo de cinco a oito dias que se seguiram ao procedimento cirúrgico, totalizando 20 dias de utilização da medicação/placebo.

Todos os pacientes foram submetidos a tratamento cirúrgico eletivo por técnica que envolveu esternotomia mediana transesternal, com introdução de cânulas na aorta e única para cava, nessa sequência. A circulação extracorpórea foi realizada com hipotermia central moderada $\left(32^{\circ} \mathrm{C}-34^{\circ} \mathrm{C}\right)$, e a proteção miocárdica foi instituída por meio de cardioplegia sanguínea hipotérmica $\left(4^{\circ} \mathrm{C}\right)$, infundida na raiz da aorta ou diretamente no seio coronariano. Todos os pacientes receberam anestesia geral balanceada, venoinalatória em circuito fechado, com absorvedor de $\mathrm{CO}_{2}$ (Cal-Sodada) e ventilação mecânica. Como agente inalatório foi utilizado o isofluorane com oxigênio a 50\%, misturado com óxido nitroso. Das drogas venosas, foram utilizados hipnóticos ethomidato e midazolam; como opioide, o fentanil; e como relaxante muscular, o brometo de pancurônio.

Para determinar a injúria pela reperfusão foram dosadas como marcadores a creatinofosfoquinase fração $\mathrm{Mb}$ e a troponina T nos seguintes intervalos: no pré-operatório, sem utilização de droga; cinco minutos após o desclampeamento aórtico, já com 12 a 15 dias de tratamento; e 12, 24 e 48 horas do pós-operatório, estando os pacientes utilizando nesse intervalo a medicação/placebo entre 15 e 18 dias. As amostras de sangue utilizadas foram obtidas mediante coleta atrial direta no desclampeamento aórtico e posteriormente em cateter posicionado no átrio direito. A CPK-Mb foi analisada pelo Olympus AU 400 ${ }^{\circledR}$, com a técnica de Szasz modificada, e a troponina $\mathrm{T}$, pelo método quantitativo imunológico, com leitura óptica através do aparelho cardiac reader ${ }^{11,15}$.

As análises estatísticas foram realizadas no software SAS 6.04 (SAS Institute, Inc., Cary, NC), utilizando-se os seguintes métodos: para a comparação das variáveis numéricas entre os dois grupos foi utilizado o teste $t$ de Student ou o teste de Mann-Whitney. Na comparação de variáveis categóricas entre os grupos foi aplicado o teste Qui-quadrado $\left(\chi^{2}\right)$ ou exato de Fisher. Para analisar o comportamento das variáveis ao longo do tempo por grupo de tratamento, foi realizada análise de variância (ANOVA) para medidas repetidas. O teste de comparações múltiplas de Bonferroni foi aplicado para identificar os momentos que diferiam entre si e entre os grupos.

O estudo foi aprovado pelo Comitê de Ética em Pesquisa do IECAC-RJ. Os participantes assinaram o termo de consentimento livre e esclarecido e foram acompanhados até a alta hospitalar.

A droga e o material de pesquisa foram custeados pelo autor. A randomização, o mascaramento e a utilização do software foram doação da Empresa Farmacopa Ltda.

\section{Resultados}

\section{Perfil geral da amostra}

A tabela 1 apresenta o perfil geral dos 60 pacientes que concluíram o ensaio clínico, comparando os grupos placebo ou controle, e aquele tratado com trimetazidina, deste ponto em diante denominados grupo placebo e grupo Tmz, respectivamente.

A tabela 2 compara a medicação utilizada pelos pacientes quando da inclusão no estudo até a cirurgia, sendo reintroduzida após a alta da Unidade de Terapia Intensiva (UTI). Os dados revelam ausência de diferença

Tabela 1 - Perfil geral da amostra segundo o grupo (Tmz e Placebo)

\begin{tabular}{|c|c|c|c|c|c|c|c|}
\hline \multirow[t]{2}{*}{ Variável } & \multicolumn{3}{|c|}{$\begin{array}{c}\text { Grupo TMZ } \\
(n=30)\end{array}$} & \multicolumn{3}{|c|}{$\begin{array}{l}\text { Grupo Placebo } \\
\qquad(n=30)\end{array}$} & \multirow[t]{2}{*}{$\mathrm{p}$ valor } \\
\hline & $\mathrm{n}$ & média $\pm \mathrm{DP}$ & $\%$ & $\mathrm{n}$ & média $\pm \mathrm{DP}$ & $\%$ & \\
\hline Sexo masculino & 19 & - & 63,3 & 24 & - & 80,0 & $0,15^{*}$ \\
\hline Idade (anos) & - & $59,8 \pm 8,1$ & - & - & $61,2 \pm 6,7$ & - & 0,46 \\
\hline Doença triarterial & 21 & - & 70,0 & 19 & - & 63,3 & 0,58 \\
\hline Diabete melito & 7 & - & 23,3 & 2 & - & 6,7 & 0,073 \\
\hline HAS & 21 & - & 70,0 & 18 & - & 60,0 & 0,41 \\
\hline IM prévio & 5 & - & 16,7 & 9 & - & 30,0 & 0,22 \\
\hline Obesidade & 3 & - & 10,0 & 2 & - & 6,7 & 0,50 \\
\hline Tabagismo & 19 & - & 63,3 & 13 & - & 43,3 & 0,12 \\
\hline FE (Simpson) & - & $59,9 \pm 8,2$ & - & - & $63,5 \pm 7,6$ & - & $0,084^{*}$ \\
\hline ICS & - & $1,1 \pm 0,15$ & - & - & $1,05 \pm 0,08$ & - & $0,19 \dagger$ \\
\hline TEI & - & $0,36 \pm 0,10$ & - & - & $0,36 \pm 0,09$ & - & $0,82^{*}$ \\
\hline
\end{tabular}

DP - desvio padrão; HAS - hipertensão arterial; IM - infarto do miocárdio; IR - insuficiência renal; FE - fração de ejeção; ICS - índice de contração segmentar (disfunção leve do ventrículo < 1,60); * teste $t$ de Student para amostras independentes; † teste de Mann-Whitney. 
Tabela 2 - Descritiva geral da medicação segundo o grupo (Placebo e Tmz)

\begin{tabular}{lccccc}
\hline \multirow{2}{*}{ Variável } & \multicolumn{2}{c}{$\begin{array}{c}\text { Grupo Placebo } \\
(\mathbf{n}=\mathbf{3 0})\end{array}$} & \multicolumn{2}{c}{$\begin{array}{c}\text { Grupo Tmz } \\
(\mathbf{n}=\mathbf{3 0})\end{array}$} & p valor* \\
\cline { 2 - 5 } & $\mathbf{n}$ & $\%$ & $\mathbf{n}$ & $\%$ & \\
\hline BBLOQ & 24 & 80,0 & 26 & 86,7 & 0,48 \\
\hline IECA & 11 & 36,7 & 13 & 43,3 & 0,59 \\
\hline BRA & 2 & 6,7 & 3 & 10,0 & 0,50 \\
\hline Nitrato & 30 & 100 & 30 & 100 & NS \\
\hline Antiagrente & 29 & 96,7 & 30 & 100 & 0,50 \\
\hline Estatina & 25 & 83,3 & 28 & 93,3 & 0,21 \\
\hline Diltiazen & 4 & 13,3 & 2 & 6,7 & 0,33 \\
\hline Diurético & 5 & 16,7 & 6 & 20,0 & 0,73 \\
\hline Hipoglicemiante & 4 & 13,3 & 5 & 16,7 & 0,71 \\
\hline
\end{tabular}

*teste de $X^{2}$ ou exato de Fisher. NS - não significativo porque são exatamente iguais; $B B L O Q$ - betabloqueador; IECA - inibidor da enzima conversora; $B R A$ - bloqueador do sistema renina-angiotensina.

significativa entre os dois grupos. Essa uniformidade evidencia especialmente que o efeito da Tmz observado no grupo tratado não foi influenciado por qualquer medicação previamente utilizada.

Comparando as variáveis ecocardiográficas da função ventricular pelos três métodos conjuntamente (ICS, Simpson e Tei), observou-se homogeneidade entre o grupo tratado e o grupo placebo no que diz respeito aos pacientes com ventrículo normal (ICS $=1$ ) e ventrículo com disfunção leve (ICS $=1,1$ a 1,6). Para fins de comparação da uniformidade da função ventricular, os pacientes foram divididos, conforme mostrado na tabela 3, em grupo sem alteração da contração segmentar (SAS) e com alteração da contração segmentar (CAS), mostrando ausência de diferença significativa da função sistólica do ventrículo entre os grupos.
Tabela 3 - Análise das variáveis basais pelo índice de contração segmentar nos grupos Placebo e Tmz

\begin{tabular}{lcccccc}
\hline \multirow{2}{*}{ Variável } & Categoria & \multicolumn{2}{c}{$\begin{array}{c}\text { Grupo } \\
\text { Placebo } \\
(\mathrm{n}=30)\end{array}$} & \multicolumn{2}{c}{$\begin{array}{c}\text { Grupo Tmz } \\
(\mathrm{n}=30)\end{array}$} & p valor \\
\cline { 2 - 5 } & & $\mathrm{n}$ & $\%$ & $\mathrm{n}$ & $\%$ & \\
\hline \multirow{2}{*}{ Subgrupo } & SAS & 17 & 56,7 & 14 & 46,7 & \multirow{2}{*}{0,43} \\
\cline { 2 - 6 } & CAS & 13 & 43,3 & 16 & 53,3 & \\
\hline Sexo & Masculino & 24 & 80,0 & 19 & 63,3 & 0,15 \\
\hline
\end{tabular}

SAS - sem alteração na contração segmentar; CAS - com alteração na contração segmentar.

\section{Análise das variáveis cirúrgicas}

A tabela 4 mostra a uniformidade global do procedimento cirúrgico, realizado por cinco equipes distintas, incluindo variáveis com elevado valor prognóstico como o tempo de anóxia, seguido pelo tempo de circulação extracorpórea, volume de solução cardioplégica, número de anastomoses e permanência média na UTI. Essa verificação coloca os dois grupos em condições cirúrgicas bastante semelhantes.

\section{Análise das variáveis ecocardiográficas}

$\mathrm{Na}$ avaliação das medidas ecocardiográficas conjuntamente entre os grupos, verificou-se que não houve diferença significativa na fração de ejeção pelo método de Simpson, pelo índice de Tei e pelo índice de contração segmentar. Observase, conforme mostrado na tabela 5, que entre cada grupo, em cada momento, e na interação entre os grupos ao longo do tempo, não houve diferença significativa. No pré-operatório sem a droga (D0), no dia da cirurgia já utilizando de 12 a 15 dias da medicação/placebo (D1), e na alta do pós-operatório com 15 a 18 dias de medicação/placebo (D2), não foi observada diferença significativa da função ventricular entre os grupos, os quais, vale notar, apresentavam uma distribuição homogênea quando analisados como subgrupos CAS e SAS (tab. 3).

Tabela 4 - Análise das variáveis cirúrgicas segundo o grupo (Placebo e Tmz)

\begin{tabular}{|c|c|c|c|c|c|c|c|c|}
\hline Variável & Grupo* & $n$ & Média & $\mathrm{DP}$ & Mediana & Mínimo & Máximo & $p$ valor \\
\hline \multirow{2}{*}{ Idade } & 1 & 30 & 61,2 & 6,7 & 62 & 46 & 72 & \multirow{2}{*}{0,46} \\
\hline & 2 & 30 & 59,8 & 8,1 & 58,5 & 47 & 80 & \\
\hline \multirow{2}{*}{ Tempo de anóxia } & 1 & 30 & 63,1 & 26,1 & 55 & 30 & 156 & \multirow{2}{*}{0,29} \\
\hline & 2 & 30 & 65,9 & 20,8 & 60 & 34 & 120 & \\
\hline \multirow{2}{*}{ Tempo de CEC } & 1 & 30 & 81,6 & 27,2 & 78,5 & 45 & 179 & \multirow{2}{*}{0,95} \\
\hline & 2 & 30 & 78,6 & 22,0 & 79,5 & 40 & 130 & \\
\hline \multirow{2}{*}{ Volume cardioplegia } & 1 & 30 & 338,3 & 171,6 & 250,0 & 200 & 750 & \multirow{2}{*}{0,76} \\
\hline & 2 & 30 & 325,7 & 120,1 & 300,0 & 200 & 600 & \\
\hline \multirow{2}{*}{ № de pontes } & 1 & 30 & 3,03 & 0,67 & 3,0 & 2 & 4 & \multirow{2}{*}{0,43} \\
\hline & 2 & 30 & 2,90 & 0,66 & 3,0 & 2 & 4 & \\
\hline \multirow{2}{*}{ Horas no CTI } & 1 & 30 & 76,8 & 13,1 & 72 & 60 & 120 & \multirow{2}{*}{0,76} \\
\hline & 2 & 30 & 74,8 & 8,7 & 72 & 60 & 96 & \\
\hline
\end{tabular}

*: 1 - grupo Placebo, e 2 - grupo Tmz; CEC - circulação extracorpórea; DP - desvio padrão. 
Tabela 5 - Análise comparativa entre os grupos Placebo e Tmz das variáveis ecocardiográficas

\begin{tabular}{lccc}
\hline \multirow{2}{*}{ Variável } & \multicolumn{3}{c}{ Componentes ( $\mathbf{p}$ valor*) } \\
\cline { 2 - 4 } & $\begin{array}{c}\text { Grupo } \\
\text { (Placebo e Tmz) }\end{array}$ & $\begin{array}{c}\text { Tempo } \\
\text { (D0, D1 e D2) }\end{array}$ & $\begin{array}{c}\text { Interação } \\
\text { (grupo*tempo) }\end{array}$ \\
\hline FE (Simpson) & 0,30 & 0,16 & 0,10 \\
\hline Tei & 0,61 & 0,63 & 0,28 \\
\hline ICS & 0,11 & 0,91 & 0,53 \\
\hline
\end{tabular}

* : ANOVA para medidas repetidas; D0 - pré-operatório sem trimetazidina; D1 - dia da cirurgia; D2 - alta do pós-operatório.

\section{Análise dos marcadores enzimáticos}

Na análise por grupo das medidas enzimáticas, os níveis da troponina T (gráfico 1) e da CPK-MB (gráfico 2) mostraram- se significativamente inferiores no grupo Tmz em relação ao grupo Placebo ( $p=0,0001)$. O mesmo ocorreu entre os grupos na evolução desses marcadores quando analisados ao longo do tempo $(p=0,0001)$. Esses níveis inferiores dos marcadores evidenciam uma maior proteção ao miocário, logo, à célula cardíaca.

\section{Discussão}

A injúria de reperfusão pode ter como manifestação funcional do ventrículo o denominado "miocárdio atordoado". Descrito por Bolli e Marban ${ }^{16}$, é basicamente um acontecimento pós-isquêmico que se caracteriza por uma depressão transitória da função do miocárdio afetado pela reperfusão, sendo esse em geral resgatado antes que o dano irreversível se instale. Para que esse dano não ocorra é necessário que a isquemia seja transitória, que a disfunção
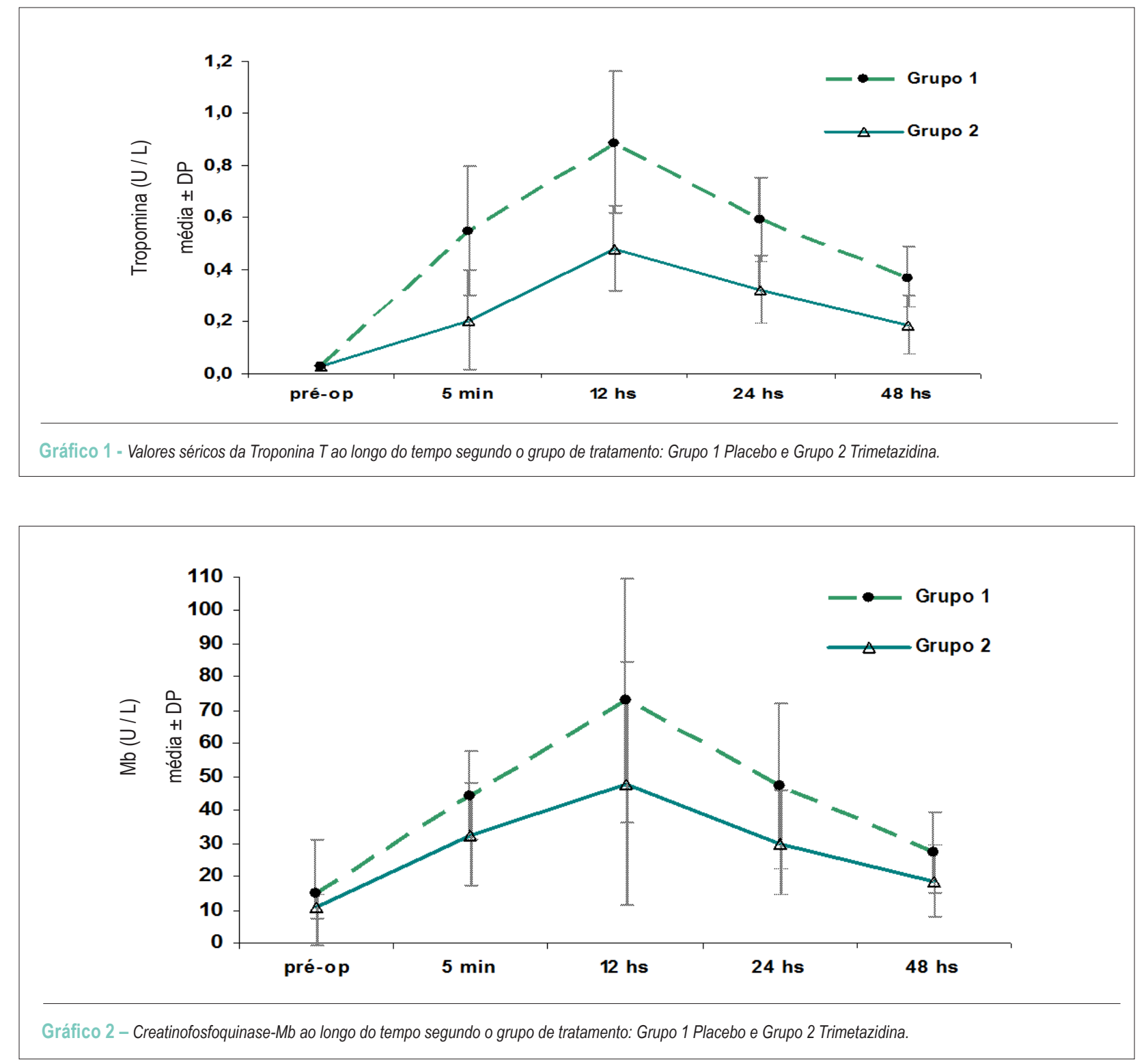
ventricular pós-isquêmica seja reversível com o tempo, e que o miocárdio receba fluxo normal ou quase normal após o episódio isquêmico ${ }^{17}$.

Os sessenta pacientes estudados, coronariopatas com lesões bi e trivasculares com função ventricular normal ou levemente comprometida, foram avaliados por três métodos ecocardiográficos em protocolo prospectivo que teve como objetivo examinar efeitos da droga trimetazidina na proteção da agressão da célula cardíaca e da função sistólica ventricular após a reperfusão em CRVM. A droga teve a posologia preconizada de $60 \mathrm{mg}$ de substância ativa, e o tempo de utilização até a cirurgia foi de no mínimo 12 dias, sendo instituída de acordo com o mecanismo de biodisponibilidade, tal como verificado em outros ensaios ${ }^{4,18}$.

O ecocardiograma transtorácico foi utilizado para analisar a função ventricular esquerda mediante métodos considerados de boa acurácia para mensurar a fração de ejeção ${ }^{12,14}$. Como método adicional foi utilizado o índice de TEI, a fim de buscar de modo não invasivo outras evidências sobre o desempenho ventricular ${ }^{19}$.

$\mathrm{Na}$ análise dos resultados não foi observada diferença significativa entre os grupos estudados pela curva da fração de ejeção por nenhum dos três métodos utilizados. Esse fato pode estar relacionado com uma limitação do método visto que a variação da função ventricular pode ocorrer logo após ao desclampeamento aórtico, caracterizando o atordoamento miocárdico de reperfusão.

Em relação aos ensaios que avaliam a função ventricular por medidas invasivas e não invasivas em CRVM de modo contínuo, foi observada melhora na função ventricular com a utilização da $\mathrm{Tmz}^{7,20-22}$. Vale ressaltar, no entanto, que neste estudo o intervalo de avaliação realizado entre o primeiro ecocardiograma e o último foi de no mínimo 48 horas, permitindo verificar apenas alterações mais persistentes na função ventricular e reduzindo assim as probabilidades de detecção do miocárdio atordoado.

Em estudos invasivos o comprometimento da função ventricular foi analisado ao término do ato cirúrgico para a injúria aguda, ou seja, no momento do desclampeamento da aorta, evidenciando de forma direta a melhoria pelo efeito da $\mathrm{Tmz}^{7,20}$. Lopatin e Dronova ${ }^{22}$ analisaram por um período de três anos 300 pacientes submetidos a CRVM, metade em pré-tratamento com Tmz e metade utilizando placebo. Os autores avaliaram a injúria de reperfusão com enzima cardíaca no pós-operatório, e durante todo o seguimento realizaram avaliações periódicas da função ventricular pela ecocardiografia e pelo teste de tolerância ao exercício. Como resultado imediato do pré-tratamento, observaram uma diminuição nos valores das enzimas cardíacas no grupo tratado em relação ao grupo placebo. Em longo prazo, foi observada diferença nos parâmetros clínicos entre os grupos, demonstrada pela melhora da classe funcional, da fração de ejeção e da tolerância ao exercício no grupo tratado.

Embora a Tmz esteja formalmente indicada no tratamento da angina estável associado ou não a drogas usuais, por apresentar pouca interação e baixa prevalência de efeitos adversos, ainda tem como característica não alterar as variáveis hemodinâmicas (frequência cardíaca e pressão arterial) sendo segura quando associada a nitratos e betabloqueadores. Nas diretrizes nacionais e internacionais, a droga tem grau de recomendação II e nível de evidência b. No entanto, neste ensaio fazemos uma utilização preventiva da droga com o objetivo de reduzir os efeitos da injuria de reperfusão pelos radicais hiperóxidos liberados na CRVM ${ }^{23}$.

Ensaios utilizando Tmz com diferentes delineamentos ligados primariamente ao tratamento clínico e que utilizaram a ecocardiografia para verificar o desempenho ventricular, com métodos semelhantes aos usados neste estudo, tais como avaliação da fração de ejeção do ventrículo esquerdo, volume sistólico final, volume diastólico final ${ }^{21}$, fração de ejeção pelo método de Simpson, escore de contração por 16 segmentos $^{24}$, e avaliação da função ventricular através do ecocardiograma de estresse com dobutamina ${ }^{25,26}$, demostraram que na maioria deles a Tmz trouxe benefícios clínicos evidenciados pela melhora na classe funcional e nos parâmetros ecocardiográficos da função ventricular.

Em relação a dosagem dos níveis de CPK-Mb e troponina $\mathrm{T}$, os valores foram significativamente inferiores no grupo submetido ao pré-tratamento com Tmz em relação grupo placebo $(p=0,001)$, sugerindo a redução da agressão pela reperfusão sobre o miocárdio.

Utilizando marcadores sorológicos de injúria miocárdica nas últimas décadas, verificou-se que os níveis séricos dessas enzimas tornaram-se instrumentos seguros para excluir ou diagnosticar o dano miocárdico. A troponina T, uma proteína miofibrilar presente no aparato contrátil, tem revelado boa acurácia por sua elevada sensibilidade quando se procura avaliar pequenas agressões ao miocárdio ${ }^{27,28}$.

Os biomarcadores ao longo do tempo vêm sendo utilizados como critérios que facilitam a identificação com precisão da injúria miocárdica de pós-operatório ${ }^{29-31}$. A troponina $t$, a troponina I e a CPK-Mb têm se mostrado úteis para definir a injúria de reperfusão, e ainda associadas a outros achados, tais como alterações eletrocardiográficas e ecocardiográficas ${ }^{15,32}$.

Comparando resultados obtidos neste ensaio com outros que utilizaram metodologia semelhante $\mathrm{e}^{7,20}$ mensurando CPK-Mb, mioglobina e troponina em pacientes submetidos a CRVM com circulação extracorpórea, observa-se que o pré-tratamento com a droga apresenta efeito cardioprotetor semelhante, que pode ser verificado mediante valores dos marcadores séricos reduzidos no grupo tratado com a Tmz.

Quando se tem como foco a proteção miocárdica relacionada à CRVM, alguns autores em estudos retrospectivos, como Klatte e cols. ${ }^{33}$ em uma das fases do estudo multicêntrico GUARDIAN, sugerem que os pacientes que evoluíram com níveis da isoenzima CPK-Mb mais elevados, com e sem relação ao surgimento de novas ondas Q com características de necrose no eletrocardiograma, apresentavam maior mortalidade no pós-operatório imediato e tardio no intervalo de seis meses.

Costa e cols. ${ }^{34}$, no estudo multicêntrico ARTS, evidenciam também que níveis mais elevados da CPK-Mb no pósoperatório de CRVM aumentam a mortalidade. Valores da isoenzima que atingem cinco vezes o valor basal elevam a mortalidade em cerca de $5 \%$ em trinta dias, e de $10 \%$ em um ano. 
Baggish e cols. ${ }^{35}$, analisando os níveis de troponina T em pacientes submetidos a cirurgia cardíaca, concluíram que o nível de troponina T superior ou igual a 1,58 ng/mL seria um forte preditor de permanência prolongada na UTI. Cabe ressaltar que no presente estudo nenhum dos pacientes atingiu esse valor de troponina, tanto no grupo placebo quanto no grupo tratado, sendo as médias de permanência na UTI semelhantes em ambos.

Lehrke e cols. ${ }^{36}$, estudando os níveis de troponina T, consideraram que valores maiores ou iguais a $0,46 \mathrm{ng} / \mathrm{mL}$ nas 48 h de pós-operatório, revelou ser um bom preditor de mortalidade cardíaca em longo prazo, especialmente nos pacientes com tempo de anóxia superior a 65 minutos. Comparando com este estudo, pode-se sugerir que a utilização da Tmz, associada a um menor tempo de anóxia, poderia ter um impacto na mortalidade em longo prazo, visto que no grupo placebo foram observados valores de troponina T superiores a 0,46 nas $48 \mathrm{~h}$ em alguns pacientes, assim como o tempo de anóxia ultrapassou 65 minutos. Entretanto, no grupo tratado, apesar de alguns pacientes terem atingido um tempo de clampeamento aórtico superior a 65 minutos, a dosagem da troponina permaneceu inferior ao valor descrito no estudo dos autores.

Outros estudos e ensaios clínicos experimentais nessa área têm demonstrado que a Tmz exerce efeito antiisquêmico direto, limitando o acúmulo de cálcio e da acidose, a inflamação e a produção de radicais hiperóxidos após reperfusão $0^{4,18,37}$, e mais recentemente, na proteção mitocondrial contra o dano pelo estresse oxidativo ${ }^{38}$. Maridonneau-Parini e Harley ${ }^{39}$ relatam que a Tmz protege a membrana celular da agressão e do dano que seguem a liberação de radicais livres hiperóxidos. Iskesen e cols. ${ }^{4,40}$ demonstraram que a droga reduz os níveis do melonaldeído, produto da ação da peroxidação lipídica dos ácidos graxos e da degradação oxidativa da membrana celular.

As alterações na proteção celular evidenciadas nos ensaios referidos acima traduzem os efeitos da Tmz na proteção à

\section{Referências}

1. Artigou JY, Chamiot JC, Bonnefont-Rousselot D, Salloum J, Grosgogeat Y. Le traitement antioxydant dans lìschemie reperfusion myocardique. Arch Mal Couer Vaiss. 1993;86(4):105-9.

2. Brasileiro ALS. A injúria de reperfusão miocárdica. Rev Socerj. 1997;10(2):79-88.

3. Malbouisson SML, Santos ML, Auler Jr JO, Carmona MJC. Proteção miocárdica em cirurgia cardíaca. Rev Bras Anestesiol. 2005;55(5):558-74.

4. Iskesen I, Saribulbul O, Cerrahoglu M, Var A, Nazli Y, Sirin H. Trimetazidine reduces oxidative stress in cardiac surgery. Circ J. 2006;70(9):1169-73.

5. Parang P, Singh B, Arora R. Metabolic modulators for chronic cardiac ischemia. J Cardiovasc Pharmacol Ther. 2005;10(4):217-23

6. Wolff A, Rotmensch HH, Stanley WC, Ferrari R. Metabolic approaches to the treatment of ischemic heart disease: the clinician's perspective. Heart Fail Rev. 2002; 7(2):187-203.

7. Turnerir B, Çolak O, Alatas O, Besogul Y, Kural T, Aslan R. Measurement of troponin $\mathrm{T}$ to detect cardioprotective effect of trimetazidine during coronary artery bypass grafting. Ann Thorac Surg. 1999;68(6):2173-6. célula miocárdica que envolve a função da mitocôndria, fornecendo para a prática clínica resultados positivos quanto à melhora da função cardíaca demonstrados nas avaliações funcionais realizadas por diversos métodos.

\section{Conclusões}

Desta análise, concluímos que a utilização da trimetazidina administrada como pré-tratamento aos pacientes com doença coronariana sem comprometimento da função ventricular esquerda ou com leve disfunção, submetidos a CRVM com circulação extracorpórea, traz evidências da redução significativa na agressão à célula cardíaca demonstrada pelos marcadores sorológicos de injúria, a CPK-Mb e a troponina T. Verificou-se também que entre os grupos estudados não houve modificações evolutivas ecocardiográficas na função sistólica ventricular esquerda examinadas no intervalo de 48 horas no pós operatório.

\section{Agradecimentos}

Agradecemos ao farmacêutico Prof. Dr. Alexandre Lourenço Lopes a confecção do placebo e o acompanhamento do processo de mascaramento e randomização do ensaio clínico; e à psicolinguista Profa. Dra. Maria do Carmo LourençoGomes, a revisão textual deste artigo.

\section{Potencial Conflito de Interesses}

Declaro não haver conflito de interesses pertinentes.

\section{Fontes de Financiamento}

O presente estudo não teve fontes de financiamento externas.

\section{Vinculação Acadêmica}

Este artigo é parte de dissertação de Mestrado de Gerez Fernandes Martins pela Universidade Federal do Estado do Rio de Janeiro e Instituto Estadual de Cardiologia Aloysio de Castro.

8. Tepliakov AT, Dzhavadova GK, Akhemedov SD, Vecherskiī II, Vesnina ZV. Effect of trimetazidine on regressione of ischemic dysfunction of myocardium after coronary bypass surgery on beating heart. Kardiologiia. 2004;44(4):51-6.

9. Gonzales-Bertomeu V, Mosquera-Bougas A, Kaski CJ. Role of trimetazidine in management of ischeamic cardiomyopathy. Am J Cardiol. 2006;98(5A):19J-24J.

10. Fox K, Garcia MA, Ardissino D, Buszman P, Camici PG, Crea F, et al. Guidelines on the management of stable angina pectoris: executive summary: The Task Force on the Management of Stable Angina Pectoris of the European Society of Cardiology. Eur Heart J. 2006;27(11):1341-81.

11. Babuin L, Jaffe SA. Troponin: the biomarker of choice for detection of cardiac injury. Clin Chem. 2001;47(3):1191-200.

12. Lang MR, Bierig M, Devereux BR, Flachskampf FA, Foster E, Pellikka PA, et al Recommendations for chamber quantification: a report from the American Society of Echocardiography's Guidelines and Standards Committee and the Chamber Quantification Writing Group, developed in conjunction with the European Association of Echocardiography, a branch of the European Society of Cardiology. J Am Soc Echocardiogr. 2005;18(12):1440-63. 
13. Cohen J. Statistical power analysis for the behavior sciences. New York: Academic Press; 1969.

14. Cerqueira DM, Weissman JN, Dilsizian V, Jacobs AK, Kaul S, Laskey WK, et al. Standardized myocardial segmentation and nomenclature for tomographic imaging of the heart. Circulation. 2002;105(4):539-42.

15. Mair J, Dienstl F, Puschendorf B. Cardiac troponin T in the diagnosis of myocardial injury. Crit Rev Clin Lab Sci. 1992;29(1):31-57.

16. Bolli R, Marbán E. Molecular and celular mechanisms of myocardial stunning Physiol Rev. 1999;79(2):609-34.

17. Braunwald E, Kloner RA. The stunned myocardium: prolonged, postischemic ventricular dysfuncion. Circulation. 1982;66(6):1146-9.

18. Vendrinne JM, Vendrinne C, Bompard D, Lehot JJ, Boissel JP, Champsaur G, et al. Myocardial protection during coronary artery bypass graft surgery: a randomized, double-blind, placebo-controlled study with trimetazidine. Anesth Analg. 1996;82(4):712-8.

19. Proheas Martinez J, Anello Utrera H, Gonzalez-Fernandez J, Rodrigues de Armas L. Índice de Tei, nuevo indicador Doppler para el estúdio de la insuficiência cardíaca. Rev Cubana Méd. 2004;43(4):1-7.

20. Fabiani JN, Ponzio O, Emerit I, Massonet-Castel S, Paris M, Chevalier P, et al. Cardioprotective effect of trimetazidine during coronary artery graft surgery. J Cardiovasc Surg. 1992;33(4):486-91.

21. Napoli DP, Tacardi AA, Barsotti A. Long term cardioprotective action of trimetazidine and potential effect on the inflammatory process in patients with ischaemic dilated cardiomyopathy. Heart. 2005;91:161-5.

22. Lopatin IUM, Dronova EP. Clinical-pharmacoeconomic aspects of trimetazidine modified release use in patients with ischemic heart disease undergoing coronary artery bypass grafting. Kardiologiia. 2009;49(2):15-21.

23. Cesar LAM, Mansur AP, Armaganyan D, Ameno JG; Sociedade Brasileira de Cardiologia. Diretriz de angina estável. Arq Bras Cardiol. 2004;83(supl 2):1-43.

24. Rosano MCG, Vitale C, Sposato B, Mercurio G, Fini M. Trimetazidine improves left ventricular function in diabetic patients with coronary artery disease: a double-blind placebo-controlled study. Cardiovasc Diabetol. 2003;2:16.

25. Chierchia LS. Trimetazidine and left ventricular ischaemic dysfuncion: an overview of clinical evidence. Eur Heart J. 2001;3(Suppl O):O16-O20.

26. César MAL, Matias Jr W, Armaganijan D, Gimenez V, Jallad S, Del Monaco MI, et al. Trimetazidine to reverse ischemia in pacients with class I or II angina: a randomized, double-blind, placebo controlled dobutamine-atropine stress echocardiography study. Coron Artery Dis. 2007;18(4):259-63.

27. Capdevila C, Portolés M, Hernandiz A, Pallares V, Cosin J. La troponina tcomo posible marcador del dano miocárdico menor: su aplicación en el miocárdio aturdido y en la isquemia silente. Rev Esp Cardiol. 2001;54(5):580-91.
28. Lang K, Borner A, Figulla HR. Comparison of biochemical markers for detection of minimal myocardial injury: superior sensibility of cardiac troponin-T ELISA. J Intern Med. 2000;247(1):119-23.

29. Kemp M, Donovan J, Higham H, Hooper J. Biochemical markers of myocardial injury. Br J Anaesth. 2004;93(1):63-73.

30. Bardorff MM, Hallermayer K, Schroder A, Ebert C, Borgya A, Gerhardt W, et al. Improved troponin T ELISA specific for cardiac troponin T isoform: assay development and analytical and clinical validation. Clin Chem. 1997;43(3):458-66.

31. Abramov D, Abu-Tailakh M, Frieger M, Ganiel A, Tuvbin D, Wolak A. Plasma troponin levels after cardiac surgery vs after myocardial infarction. Asian Cardiovasc Thorac Ann. 2006;14(6):530-5.

32. Carrier M, Pellerin M, Perrault PL, Solymoss BC, Pelletier LC. Troponin levels in pacients with myocardial infarction after coronary artery bypass grafting. Ann Thorac Surg. 2000;69(2):435-40.

33. Klatte K, Shaidman BR, Theroux P, Gavard JA, Stocke K, Boyce S, et al. Increase mortality after coronary artery bypass grast surgery is associated whith increased levels of postoperative creatinekinase-myocardial and band isoenzime release.(GUARDIAN trials). J Am Coll Cardiol. $2001 ; 38(4): 1070-7$.

34. Costa MA, Carere RG, Lichtenstein SV, Foley DP, de Valk V, Lindenboom W, et al. Incidence, predictors and significance of abnormal cardiac enzyme rise in patients treated with bypass surgery in the arterial revascularization therapies study (ARTS). Circulation. 2001;104(22):2689-93.

35. Baggish LA, MacGillivray ET, Hoffman W, Newell JB, Lewandrowski KB, LeeLewandrowski E, etal. Postoperative troponin-t predicts prolonged intensive care unit lenght of stay following cardiac surgery. Crit Care Med. 2004;34(9):1866-71.

36. Lehrke S, Steen H, Sievers HH, Peters H, Opitz A, Müller-Bardorff M, et al. Cardiac troponin $\mathrm{T}$ for prediction of short-and long-term morbidity and mortality after elective open heart surgery. Clin Chem. 2004;50(9):1560-7.

37. Renauld JF. Internal $\mathrm{pH}, \mathrm{Na}^{+}$and $\mathrm{Ca}^{2+}$ regulation by trimetazidine during cardiac cell acidosis. Cardiovasc Drugs Ther. 1988;1(6):677-86.

38. Argaud L, Gomes L, Gateau-Roesch O, Couture-Lepetit E, Loufouat J, Robert D, et al. Trimetazidine inhibits mitochondrial permeability transition pore opening and prevents lethal ischemia-reperfusion injury. J Mol Cell Cardiol. 2005;39(6):893-9.

39. Maridonneau-Parini I, Harpey C. Effect of trimetazidine on menbrane damage induced by oxygen free radicals in human red cells. $\mathrm{Br} J$ Clin Pharmacol. 1985;20(2):148-51.

40. Iskesen I, Kurdal AT, Eserdag M, Cerrahoglu M, Sirin BH. Trimetazidine may protect the myocardium during cardiac surgery. Heart Surg Forum. 2009;12(3):E175-9. 